\title{
Blood glucose control in a pregnant female with Type 1 diabetes and Medium- chain acyl-CoA dehydrogenase deficiency (MCADD)
}

\author{
Wilson $D^{1}$, Brown $A^{1}$, Gumma $A^{2}$, Oyibo $S^{1}$ \\ ${ }^{1}$ Department of Diabetes \& Endocrinology and the ${ }^{2}$ Department of Obstetrics \& Gynaecology, \\ Peterborough City Hospital, Peterborough, UK
}

\section{Background}

Medium-chain acyl-CoA dehydrogenase deficiency (MCADD) is an autosomal recessive inherited disorder resulting in the inability to breakdown medium-chain fatty acids to provide energy during periods of lowcalorie intake and hypoglycaemia (infections, fasting, vomiting). Without urgent treatment (intravenous $10 \%$ glucose), the accumulation of toxic fatty acids can lead to encephalopathy and sudden death. Affected individuals are used to taking frequent glucose-containing drinks for regular energy provision.

Pregnant females with Type 1 diabetes require strict glucose control for a favourable outcome for mother and baby. National guidelines advise aiming for fasting capillary blood glucose (CBG) levels $<5.3 \mathrm{mmol} / \mathrm{l}$, pre-meal CBG 4-6 mmol/l and 1-hour post-meal CBG levels $<7.8 \mathrm{mmol} / \mathrm{l}$, while avoiding hypoglycaemia.

We present a case combining these two conditions in pregnancy.

\section{Case}

A 17-year-old lady was diagnosed with MCADD soon after birth and Type 1 diabetes at age 7 years. She had paediatric/metabolic disease specialist care throughout her childhood and kept her CBG levels raised to avoid hypoglycaemia [median (range) HbA1c: 89 (68-129) $\mathrm{mmol} / \mathrm{mol}$, over the past 3 years]. She attended our adult diabetes-antenatal clinic during her pregnancy. She has a home protocol and we have an emergency protocol for treatment during acute illness/decompensation.

\section{Management}

With 1-2 weekly clinic visits she continued her basal-bolus insulin regimen (Lantus \& Novorapid), Folic acid and Aspirin. She improved her CBG levels throughout pregnancy: median (range) HbA1c: 53 (42-55) $\mathrm{mmol} / \mathrm{mol}$. During labour and delivery we used a variable rate insulin and glucose infusion, aiming for CBG levels between $5-10 \mathrm{mmol} / \mathrm{l}$ rather than the usual $4-7.8 \mathrm{mmol} / \mathrm{l}$, so as to maintain sufficient glucose-related energy and block alternative energy-producing pathways. She had an uncomplicated delivery.

Figure 1 - Medium-chain acyl-CoA dehydrogenase deficiency (MCADD)

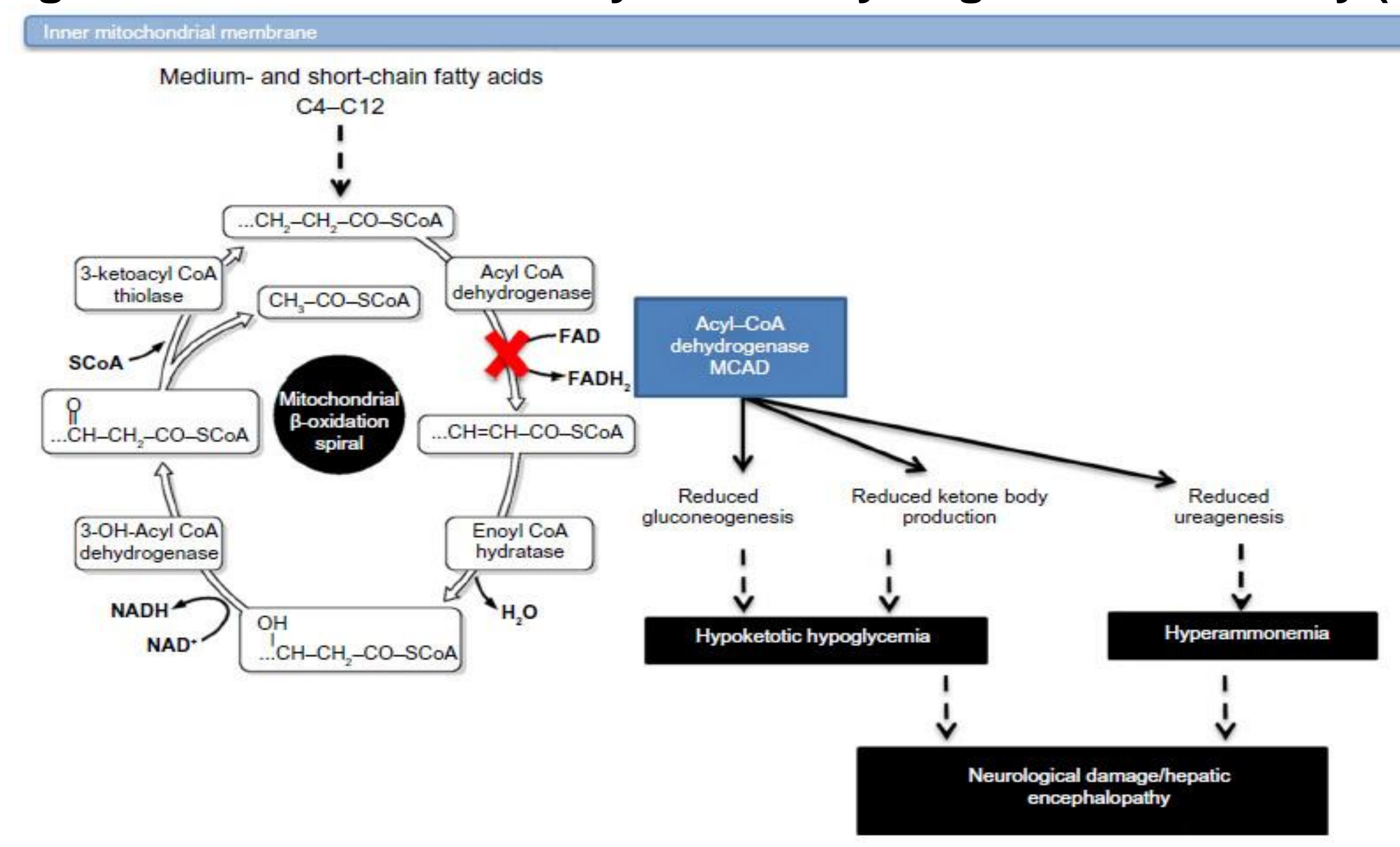

\section{Conclusion}

The combination of Type 1 diabetes and MCADD in pregnancy is a rare challenge to the obstetrician, diabetologist, dietitian and most importantly, to the patient. Uncomplicated cases (with no concurrent illness, frequent vomiting or prolonged fasting) should go well. The importance of a multidisciplinary team approach to care and seeking advice from the inherited metabolic disease specialist cannot be overemphasized.

1. MCAD Deficiency Guidelines. British Inherited Metabolic Disease Group (revised 2017). http://bimdg.org.uk

2. Bashari WA, Oyibo SO. Inborn errors of metabolism and the endocrinologist. Endocrine Abstracts $2015 ; 38$ : P281. 